\title{
Cerebellar ataxia as presenting feature of hypothyroidism
}

Suman Kumar Kotwal', Shalija Kotwal', Rohan Gupta', Jang Bhadur Singh', Annil Mahajan'

\section{SUMMARY}

Symptoms and signs of the hypothyroidism vary in relation to the magnitude and acuteness of the thyroid hormone deficiency. The usual clinical features are constipation, fatigue, cold intolerance and weight gain. Rarely it can present with neurologic problems like reversible cerebellar ataxia, dementia, peripheral neuropathy, psychosis and coma. Hypothyroidism should be suspected in all cases of ataxia, as it is easily treatable. A 40 year-old male presented with the history facial puffiness, hoarseness of voice and gait-ataxia. Investigations revealed frank primary hypothyroidism. Anti-TPO antibody was positive. Thyroxine was started and patient improved completely within eight weeks. Hypothyroidism can present with ataxia as presenting feature. Hypothyroidism should be considered in all cases of cerebellar ataxia as it is a reversible cause of ataxia. Arch Endocrinol Metab. 2016;60(2):183-5

\author{
1 Department of Medicine, \\ Government Medical \\ College, Jammu, India \\ 2 Department of Pathology \\ Government Medical \\ College, Jammu, India \\ Correspondence to: \\ Suman Kumar Kotwal \\ Department of Medicine, \\ Government Medical College, \\ Jammu, India \\ sumankk1230@rediffmail.com \\ Received on July/07/2014 \\ Accepted on Sept/10/2015
}

DOI: 10.1590/2359-3997000000121

\section{INTRODUCTION}

$\mathrm{H}$ ypothyroidism is a common medical condition in the general population. Common systemic manifestations include fatigue, constipation, cold intolerance, weight gain, hair loss, dry skin, and hoarseness. A variety of central and peripheral nervous system manifestations are common in patients with hypothyroidism. In many cases, the neurologic manifestations occur in conjunction with the systemic features of the disease and may be noted only incidentally. However, symptoms and signs of neurologic dysfunction may develop in some patients and can contribute to significant disability. Most of these complications are partially or fully responsive to thyroxine replacement (1). There is scarce recent published data on hypothyroidism causing ataxia. Here we present a case of hypothyroidism with cerebellar ataxia.

\section{CASE REPORT}

We present a case of a 40 -year-old male who reported to us with history of instability of gait since 3 weeks which was acute in onset and progressive, there was also history of puffiness, hoarseness of voice and constipation from few months. There was no history of weakness in any part of body, headache, vomiting, convulsions or alteration of sensorium. There was no history of trauma to the head, fever or drug intake. On examination his vitals were normal. Cognitive functions were normal. Neurological examination showed gait ataxia, dysarthria and dysmetria on finger-nose and heel-to-knee tests. There was horizontal nystagmus. The gait was wide-based and there was a tendency to fall to right side. His fundus was normal. His power was normal but had hung up reflexes. Sensory system was normal. His hemogram was normal. Serum electrolytes, blood sugar, renal and liver function tests were normal. Serum CPK (creatine phosphokinase): $1220 \mathrm{U} / \mathrm{L}$. Total cholesterol: $225 \mathrm{mg} / \mathrm{dL}$, triglycerides: $275 \mathrm{mg} / \mathrm{dL}, \mathrm{HDL}$ cholesterol: $36 \mathrm{mg} / \mathrm{dL}$, LDL cholesterol: $150.20 \mathrm{mg} /$ dL, VLDL cholesterol: $35 \mathrm{mg} / \mathrm{dL}$. T3: 60 (80-200 $\mathrm{ng} / \mathrm{dL}) \mathrm{T} 4: 2.5 \mathrm{ug} / \mathrm{dL}(4.5-12.5)$, TSH: $88 \mathrm{uIU} / \mathrm{mL}$ (0.27-5.5). The Serum anti-TPO antibody was elevated $(500 \mathrm{IU} / 1$; reference: < 34). His chest X-ray was normal. MRI brain was normal (Figures $\mathrm{l}$ and 2). $\mathrm{He}$ was started on $75 \mu \mathrm{g}$ of thyroxine. TSH after 2 months of therapy was $2.8 \mathrm{uIU} / \mathrm{mL}$. His lipid profile and CPK also normalized. He was completely normal after few weeks. 




Figure 1. T1 weighted MRI showing normal cerebellum.

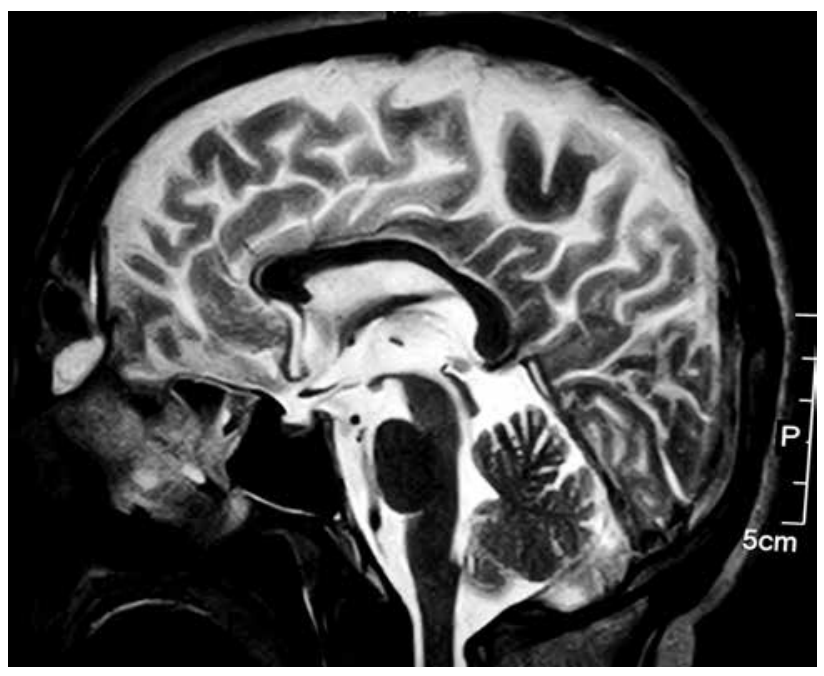

Figure 2. T2 weighted MRI brain showing normal cerebellum.

\section{DISCUSSION}

Hypothyroidism is one of the causes of acute onset ataxia. Stroke, viral encephalitis and drugs can also cause acute cerebellar ataxia. Mass lesions in the posterior fossa, infections such as HIV, and vitamin deficiencies like $\mathrm{Bl}$ and B12, alcohol and paraneoplastic syndromes are causes of sub acute onset cerebellar ataxia in an adult. Hypothyroidism has been recognized as a cause of gait ataxia (2).

In early case series of patients with adult-onset hypothyroidism, a wide-based gait ataxia was a prominent feature in a considerable number, around 10 to $30 \%$ of cases $(1,3)$. In many cases, this was the presenting feature, and variably included clumsiness, intention tremor, dysmetria and dysarthria (4). Treatment of the hypothyroid state led to improvement or resolution of the cerebellar features.

Cerebellar dysfunction is also a feature of congenital hypothyroidism. Up to 40 percent of children and adolescents with congenital hypothyroidism have been shown to demonstrate mild findings of cerebellar dysfunction, such as intention tremor, difficulty with tandem gait, clumsy running, fine motor dysfunction, and unsteady stance (5). Animal studies have shown that hypothyroidism interferes with cerebellar development (6). The cause of cerebellar ataxia in association with hypothyroidism remains unidentified. No typical morphological changes in the cerebellum have been described. It is assumed that a thyrogenic, specific metabolic factor is responsible which aggravates already existing non specific cerebellar changes. The prognosis is very good. On the other hand, cerebellar ataxia resulting from congenital hypothyroidism has typical histological cerebellar changes and prognosis is very poor unless thyroxine treatment is started soon after birth (7).

Cerebellar degeneration in patients with raised antithyroid antibodies may be immune mediated. Some cases of hypothyroidism associated cerebellar ataxia do not reverse with normalization of their thyroid state with thyroid hormone replacement and may be harboring undiagnosed Hashimoto's thyroiditis (8). Dinkar and cols. have reported a case of Hashimoto's encephalopathy presenting purely with sub acute cerebellar syndrome (9). A similar case has been reported by $\mathrm{Na}-$ kagawa and cols. (10). Necropsies of patients with hypothyroidism and cerebellar ataxia have been reported. Barnard and cols. described degenerative changes in the cerebellum, particularly in the anterosuperior portion of the vermis, together with atrophy of ventral portion of the pons, transverse pontine fibres, and middle and superior peduncles in a patient with hypothyroidism and cerebellar ataxia (2).

In conclusion, hypothyroidism should be considered in all cases of cerebellar ataxia, as it is a reversible cause of ataxia.

Acknowledgements: none declared.

Disclosure: no potential conflict of interest relevant to this article was reported. 


\section{REFERENCES}

1. Sandyk R. Cerebellar dysfunction in hypothyroidism. S Afr Med J. 1982;62(14):468.

2. Barnard RO, Campbell MJ, McDonald WI. Pathological findings in a case of hypothyroidism with ataxia. J Neurol Neurosurg Psychiatry. 1971;34(6):755-60.

3. Cremer GM, Goldstein NP, Paris J. Myxedema and ataxia. Neurology. 1969;19(1):37-46.

4. Quinn N, Barnard RO, Kelly RE. Cerebellar syndrome in myxoedema revisited: a published case with carcinomatosis and multiple system atrophy at necropsy. J Neurol Neurosurg Psychiatry. 1992;55(7):616-8.

5. Wiebel J. Cerebellar-ataxic syndrome in children and adolescents with hypothyroidism under treatment. Acta Paediatr Scand. 1976;65(2):201-5.
6. Koibuchi N, Jingu H, Iwasaki T, Chin WW. Current perspectives on the role of thyroid hormone in growth and development of cerebellum. Cerebellum. 2003;2(4):279-89.

7. Hammar $\mathrm{CH}$, Regli F. Cerebellar ataxia due to hypothyroidism in adults (case report). Dtsch Med Wochenschr. 1975;100(28):1504-6.

8. Selim M, Drachman DA. Ataxia associated with Hashimoto's disease: progressive non-familial adult onset cerebellar degeneration with autoimmune thyroiditis. J Neurol Neurosurg Psychiatry. 2001;71(1):81-7.

9. Dinkar K, Sinha S, Taly AB, Bindu PS, Bharath R. Steroid-responsive encephalopathy in autoimmune thyroiditis: clinical spectrum and MRI observations in three cases. Ann Indian Acad Neurol. 2011;14:205-7.

10. Nakagawa H, Yoneda M, Fujii A, Kinomoto K, Kuriyama M. Hashimoto's encephalopathy presenting with progressive cerebellar ataxia. J Neurol Neurosurg Psychiatry. 2007;78(2):196-7. 